\title{
Diacronie
}

Studi di Storia Contemporanea

$N^{\circ} 20,4 \mid 2014$

II diritto militante

\section{La scienza nel processo penale: Porto Marghera}

\section{Marina De Ghantuz Cubbe}

\section{(2) OpenEdition \\ Journals}

\section{Edizione digitale}

URL: http://journals.openedition.org/diacronie/1707

DOI: 10.4000/diacronie. 1707

ISSN: 2038-0925

\section{Editore}

Association culturelle Diacronie

Notizia bibliografica digitale

Marina De Ghantuz Cubbe, "La scienza nel processo penale: Porto Marghera », Diacronie [Online], N 20, 4 | 2014, documento 7, Messo online il 01 décembre 2014, consultato il 19 avril 2019. URL : http:// journals.openedition.org/diacronie/1707; DOI : 10.4000/diacronie.1707 


\title{
Diacronie
}

\section{La scienza nel processo penale: Porto Marghera}

\author{
Marina de GHANTUZ CUBBE*
}

Lo chiamavano Morto Marghera. Il polo sito a Mestre, accanto alla bella Venezia è stato almeno dal 1917 scenario di uno sviluppo industriale fondamentale per l'Italia. Porto Marghera ha ospitato aziende come la Montedison, che ha costruito qui la sua fortuna. La promessa di un futuro migliore anche per i lavoratori e per le famiglie che da tutta Italia vi approdavano si è trasformata nella morte di 157 operai. Le sostanze che lavoravano li avevano uccisi. La storia di Porto Marghera si sposta così nelle sentenze di un discusso processo in cui la "verità" della prova scientifica rischia di prevalere sulla giustizia. Gianfranco Bettin in un'intervista ritorna con la mente al processo: "è impossibile assolvere chi, sapendo di esporre i lavoratori a effetti letali, ha nascosto".

\section{Il polo industriale}

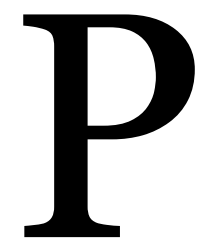

orto Marghera ha rappresentato, a partire dal 1917, anno dei primi insediamenti, una promessa di sviluppo tale da convogliare le energie e le vite di migliaia di persone. Nel 1927 Venezia deteneva, allinterno della regione Veneto, il primato nel settore meccanico, nella lavorazione dei minerali non metalliferi e nel settore chimico. «Era accaduto quel fatto significativo e davvero fondamentale nella storia dello sviluppo veneto, rappresentato da Porto Marghera». In dieci anni si era avviato il processo di industrializzazione e sviluppo della laguna pensato dall'ingegner Coen Cagli, il quale aveva elaborato un progetto

${ }^{1}$ ROVERATO, Giorgio, La terza regione industriale in LANARO, Silvio (a cura di), Storia d'Italia. Le regioni dall'Unità ad oggi. Il Veneto, Torino, Einaudi, 1984, pp. 193. 
ambizioso per la costruzione del nuovo porto di Venezia in terraferma con annessi zona industriale e quartiere urbano; «tale progetto venne dichiarato "meritevole di approvazione" dal Consiglio superiore dei LLPP [lavori pubblici] il 15 maggio 1917»². Partecipava al progetto il Sindacato di studi per imprese elettro-metallurgiche nel porto di Venezia, nato con il consenso e l'appoggio del Comune e della Provincia e presieduto dal conte Giuseppe Volpi di Misurata, Ministro delle finanze del governo Mussolini dal 1925 al 1928. Il 12 giugno 1917 il Sindacato si trasformava in Società del porto industriale di Venezia e il 23 luglio firmava la Convenzione relativa alla concessione della costruzione del nuovo porto di Venezia, in regione di Marghera, e ai provvedimenti per la zona industriale e il quartiere urbano. Mancavano due mesi alla disfatta di Caporetto e intanto a Porto Marghera iniziava un'altra storia italiana; protagonista era il Consorzio ${ }^{3}$ che ne stabiliva le sorti. I piani regolatori, realizzati dai partecipanti al Consorzio presieduto dal già citato Giuseppe Volpi, riguardavano sostanzialmente il continuo ampliamento dell'insediamento; alla $\mathrm{I}^{\mathrm{a}}$ zona industriale in cui primeggiava la SADE (Società Adriatica di Elettricità) di proprietà dello stesso Volpi, si affiancò la II ${ }^{\mathrm{a}}$ zona pensata tra il 1953 e il 1956, attraverso la quale venne realizzata un'area completamente autonoma e consacrata alla chimica. La società Edison ${ }^{4}$ dava infatti avvio all'invasione della nuova zona, insediando produzioni che integravano quelle già in atto nel cosiddetto Petrolchimico1 del 1949.

\begin{abstract}
A partire dal 1953 iniziava a disegnarsi all'interno della seconda area di espansione un gigantesco stabilimento articolato per sezioni operative, tutte coordinate da una direzione unificata, quella dell'Edison. Le vicende degli anni Cinquanta dell'Edison gettano le basi di uno sviluppo che non conoscerà battute d'arresto, grazie soprattutto alla continua modernizzazione degli impianti, sinonimo di potenziamento industriales.
\end{abstract}

\footnotetext{
${ }^{2}$ CHINELLO, Cesco, Storia di uno sviluppo capitalistico. Porto Marghera e Venezia 1951-1973, Roma, Editori Riuniti, 1975, p. 14.

3 La prima legge a istituzionalizzare la composizione del Consorzio è la n. 1233 del 20 ottobre 1960; essa conferisce piena legittimità anche alle opere realizzate precedentemente. Oltre ai proprietari delle singole industrie (insieme a G. Volpi altri otto privati), vengono nominati a contribuire economicamente: la Camera di commercio, industria e agricoltura di Venezia; il comune di Venezia; la provincia di Venezia e il Provveditorato al porto di Venezia.

$4 \mathrm{Il} 28$ giugno 1883 entrò per la prima volta in funzione in Italia la centrale termoelettrica che inaugura il servizio di distribuzione pubblica dell'energia elettrica. Con sede a Milano, l'Edison si espanse notevolmente dal primo dopoguerra in avanti anche ad altri settori, in particolare quello chimico.

5 BARIZZI, Sergio, RESINI, Daniele (a cura di), Portomarghera. Il Novecento industriale a Venezia (cd-rom), Venezia, Vianello Libri, 2004, p. 251.
} 
L'invasione diviene ancora più evidente nel 1966, anno in cui la Montecatini ${ }^{6}$ e l'Edison si fondono nella Montedison andando a detenere l'84\% delle aree di pertinenza del polo industriale. La Montedison «a ridosso degli anni Settanta copre inoltre l'80\% della produzione nazionale per quanto riguarda la chimica fine» 7 e sin dal 1971, anno di insediamento di Eugenio Cefis a capo dell'azienda, l'area del Petrolchimico è segnata da processi di ristrutturazione caratterizzati da continue espansioni. È così che durante questi anni Porto Marghera consolida definitivamente una posizione di primo ordine nello scenario dello sviluppo industriale italiano. Per tale conquistata egemonia e per altri, tragici motivi, la Montedison e Porto Marghera confluiranno, nella «memoria post-industriale» 8 , in un unico luogo rappresentativo dell'immaginario collettivo: il Petrolchimico, anche detto Petrolkiller 9.

«Risalgono agli anni Settanta i molteplici e complessi riassetti societari ma un momento di svolta in questo senso è segnato dall'affare Enimont ${ }^{10}$ » varato nel 1989 , che doveva raggruppare le attività di Montedison e di Enichem (società dell'Eni) creando un vero e proprio colosso della chimica nel mondo; il progetto fallì e il futuro di Porto Marghera divenne sempre più incerto, sino al declino. Il successore di Eugenio Cefis (1971- 1977), Raul Gardini (1986-1991), fu promotore del suddetto progetto, responsabile del fallimento, vittima e carnefice allo stesso tempo: ad un anno dall'inizio dell'inchiesta Mani Pulite in cui per l'affare Enimont erano indagati tutti i più alti dirigenti del gruppo, si suicida con un colpo di pistola.

La responsabilità del grande gruppo dirigente non è stata solo quella di aver fatto sprofondare la Montedison in una crisi autodistruttiva, peraltro pagata alla fine dal denaro pubblico oltre che dal salario e dal lavoro di migliaia di operai; ma di aver avvelenato $\mathrm{i}$ lavoratori in fabbrica e la popolazione di un vasto territorio inquinato da ogni sorta di sostanze tossiche e nocive ${ }^{11}$.

\footnotetext{
${ }^{6}$ La Montecatini nasce nel 1888 a Firenze per lo sfruttamento delle miniere di rame del posto; nel 1910 si orientò verso l'industria chimica in particolare nella produzione di fertilizzanti necessari all'agricoltura. Nel 1936 insieme allo Stato italiano fondò l'ANIC per la produzione di benzina e la raffinazione del petrolio.

7 ZUCCONI, Guido, Marghera e la scommessa industriale veneziana, in ADORNO, Salvatore, NERI SERNERI, Simone (a cura di), Industria, ambiente e territorio. Per una storia ambientale delle aree industriali in Italia, Bologna, Il Mulino, 2009, pp. 145-146.

8 CERASI, Laura, Perdonare Marghera. La città del lavoro nella memoria post-industriale, Milano, Franco Angeli, 2007.

9 BETTIN, Gianfranco, DIANESE, Maurizio, Petrolkiller. In appendice i documenti segreti delle aziende chimiche, Milano, Feltrinelli, 2002.

10 BARIZZI, Sergio, RESINI, Daniele (a cura di), Portomarghera, cit, p. 253.

${ }^{11}$ CHINELLO, Cesco, Storia di uno sviluppo capitalistico cit., p. 53.
} 
Gli operai di Porto Marghera, sin dal secondo dopoguerra hanno vissuto le vicende delle imprese attraverso scioperi e mobilitazioni. Si sono raggiunte, soprattutto durante l' "autunno caldo", non solo conquiste contrattuali:

Porto Marghera è stata una delle punte più avanzate del paese, sia per le multiformi sollecitazioni spontaneiste che si sono levate dal basso che per le forme articolate di lotta. È dentro queste tensioni e sempre dal basso che si è fatta strada, a partire dai reparti CV (Cloruro di vinile) del Petrolchimico, anche una nuova forma di contestazione-rivendicazione: "La salute non si paga"12.

\section{Il processo}

Sul finire degli anni Sessanta tra gli operai e i sindacati di Porto Marghera si sapeva poco o niente rispetto ai rischi corsi ogni giorno nei reparti di lavorazione CV. Nel 1966 la forte alluvione che sommerge Venezia preoccupava il mondo intero e le istituzioni varavano nel 1973 la prima legge speciale per Venezia; era la prima legge a non dare avvio a nuovi lavori bensì atta a difendere un patrimonio storico e ambientale. Ma la legge riguardava Venezia, non la vicina Marghera. In quegli anni infatti all'interno del polo industriale la problematica non era ancora la salvaguardia dell'ambiente né tanto meno la salute umana: «dell'ambiente nocivo e di rischio al Petrolchimico, come in tante altre fabbriche, se ne dava per scontato un certo grado come dato obbligato e lo si ripagava con una indennità salariale ai lavoratori, considerata persino una conquista» ${ }^{13}$.

Gianfranco Bettin, oggi assessore all'ambiente presso il Municipio di Mestre, ricorda con queste parole quel periodo e l'atteggiamento generale che definisce tra l'irresponsabile, il fatalistico e il temerario:

prima dell'approvazione della legge speciale, a Porto Marghera ci sono fughe di gas, incidenti, c'è questo tipo di problematica; l'incidente più che l'impatto che in sé il polo industriale può avere, produce le prime voci attorno al rischio del CVM soprattutto nella parte chimica, oppure al rischio implicito nella presenza di grandi depositi di fosgene. Io abitavo al confine con la zona alfa, quella dove la distruzione sarebbe stata totale in caso di incidente ${ }^{14}$.

12 Ibidem.

13 Ibidem.

${ }^{14}$ Intervista realizzata da DE GHANTUZ CUBBE, Marina, il 7 marzo 2014 presso il Municipio di Mestre. 
Sono voci quelle attorno al CVM (Cloruro di vinile monomero) e non riguardano ancora la pericolosità della sostanza in sé; la paura è dovuta alle fughe di gas, alle possibili esplosioni, agli incidenti sul lavoro. Anni dopo, durante una riunione sindacale nell'apposito capannone del Petrolchimico (una delle conquiste dell' “autunno caldo"), si concretizzano i timori e le voci. Ma in qualcosa che nessun operaio lontanamente sospettasse: il cancro.

La storia di Porto Marghera e della sua principale impresa è tristemente legata alla morte di 157 operai, ai danni ambientali causati dalle industrie alla laguna, ai danni fisici e morali subiti dalle famiglie dei lavoratori. Non solo: la storia della Montedison è stata scritta anche dal processo penale che l'ha riguardata per anni. Il processo a Marghera e tutto ciò che lo ha causato hanno rappresentato lo scontro tra la società civile e i personaggi che erano a capo delle imprese, Eugenio Cefis in primis; la tensione tra il diritto al lavoro e il diritto alla vita ma anche tra diritto penale, scienza e verità storica.

Le denunce che portarono al processo del 1994 furono il risultato di una lunga serie di tensioni e paure che andavano accumulandosi a Porto Marghera da più di venti anni: del 1974 è la riunione sindacale in cui ai lavoratori venne per la prima volta esplicitata la pericolosità della sostanza lavorata in alcuni stabilimenti del Petrolchimico.

Due anni prima, nel 1972, moriva Simonetto Ennio capo turno del famigerato reparto CV 14-16 in cui veniva lavorato il CVM e, a detta del professor Cesare Maltoni dipendente della Montedison, moriva di angiosarcoma epatico, raro tumore associato alla suddetta sostanza ${ }^{15}$.

Cesare Maltoni, oncologo bolognese, fu sovvenzionato dalla Montedison per approfondire le ricerche già effettuate da Luigi Viola per conto di un'altra industria, la Solvay.

Quest'ultimo era partito dall'osservazione dei troppi casi di sindrome di Raynaud riscontrati tra gli operai del CVM-PVC nello stabilimento di Rosignano nel corso dei primi anni Sessanta. Le ricerche erano state condotte sui ratti e anche Maltoni proseguì su questa strada confermando nel 1974 l'attribuzione della sindrome proprio al CVM ${ }^{16}$.

${ }^{15}$ CASSON, Felice, La fabbrica dei veleni. Storie e segreti di Porto Marghera, Milano, Sperling \& Kupfer, 2007, p. 27.

${ }^{16}$ Ibidem, p. 28. 
Tornando a Simonetto Ennio, primo caso italiano, al momento della sua morte nessuno aveva collegato l'angiosarcoma al CVM perché le ricerche di Viola non erano state rese note ai lavoratori. Ma due anni dopo Maltoni durante l'assemblea nel capannone del Petrolchimico, confermava sia tale associazione sia che le morti degli operai della società B. F. Goodrich di Louisville negli Stati Uniti erano dovute anch'esse al CVM. Due anni, ma in realtà molti di più, in cui gli altri autoclavisti come Simonetto Ennio avevano continuato a lavorare immersi in quella sostanza tossica. Con queste parole Renzo Marin, autoclavista, ricorda quel lavoro:

\begin{abstract}
Al Cv6 si produceva il Pvc, una polvere di plastica, noi dovevamo fare il carico, lo scarico e la pulizia delle autoclavi. Per pulire le incrostazioni ci facevano entrare nelle autoclavi attraverso uno stretto boccaporto, eravamo imbracati, dall'esterno un compagno ci teneva con una corda e era pronto a tirarci fuori nel caso qualcuno si sentisse male o svenisse. Si entrava tramite una scala a pioli, si scendeva di circa tre metri, l'interno era illuminato da una lampada che si agganciava alle pareti, la temperatura raggiungeva i $40^{\circ} \mathrm{C}$, le incrostazioni di plastica si formavano in particolare sulle pale dell'agitatore e sui bocchettoni di entrata dei prodotti. Bisognava toglierle con lo scalpello e battere con una certa forza. Restavi dentro fino a che riuscivi a resistere, talvolta anche per quindici minuti. Le incrostazioni emanavano una quantità impressionante di gas ${ }^{17}$.
\end{abstract}

Un altro autoclavista operaio della Montedison, era Gabriele Bortolozzo. Aveva lavorato per venticinque anni nel reparto CV-6 e nel 1982 era stato spostato al CV 1416. Mal voluto sia dai sindacalisti sia dai dirigenti fu lui, assieme a Medicina Democratica, a portare sul tavolo dell'allora Procuratore della Repubblica Felice Casson le denunce da cui partirà il processo. Proprio Felice Casson riferisce del rapporto tra sindacati e Bortolozzo, mettendo in luce la difficoltà di un singolo individuo che durante i primi anni Ottanta

aveva cominciato a protestare, duramente, anche più della Commissione ambiente del Petrolchimico e dei sindacati. Li aveva scavalcati e anche accusati di inerzia. Era entrato in conflitto con tutti coloro che comandavano in fabbrica, da una parte (i dirigenti) e dall'altra (i sindacati) ${ }^{18}$.

${ }^{17}$ SACCAROLA, Antonella, Pensando a Marghera. Il luogo e la memoria: viaggio in forma di interviste-racconti, Treviso, Alcione, 2006, p.51.

${ }_{18}$ CASSON, Felice, La fabbrica dei veleni cit., p.146. 
Difficoltà nel fare indagini, raccogliere dati e informazioni sui colleghi, tali e tanti da poter sporgere denuncia contro l'impero di Eugenio Cefis. Bortolozzo non era completamente solo, almeno non fuori dal Petrolchimico: Medicina Democratica ha, dalla sua fondazione, sempre e conformemente a quanto prevede il suo statuto, presentato esposti e denunce in relazione soprattutto al decesso di lavoratori esposti a sostanze cancerogene. Tale associazione vede il suo fondatore in Giulio Alfredo Maccacaro e

dalla fine degli anni Sessanta ad oggi si pone in continuità storica, culturale, economica, scientifica, operativa e ideale con il Movimento di Lotta per la salute. Le azioni portate avanti, tutte di carattere pacifista e non violento, vertono al pieno rispetto e alla tutela dei diritti civili e dei diritti umani. In primis il diritto alla salute (art. 32 della Costituzione) e il diritto all'ambiente (art. 9) vengono difesi ogni qual volta essi siano violati attraverso una militanza volontaria e con azioni concrete: promozione di iniziative sociali, culturali, politiche nonché con il ricorso all'Autorità Giudiziaria ${ }^{19}$.

Gabriele Bortolozzo fu informato e formato, sostenuto, supportato in ogni singola azione da Medicina Democratica e in nome di una giustizia sociale calpestata, rischiava ogni giorno di subire forti ritorsioni da parte dell'azienda. Ad accompagnare in prima persona Bortolozzo a sporgere denuncia fu proprio un esponente di Medicina Democratica, Luigi Scatturin.

\begin{abstract}
Raro esempio di professionalità e di passione civile (Casson), è stato un avvocato membro di importanti collegi di difesa nazionali, costituiti per rilevanti processi penali e civili negli anni '70/'80 patrocinando operai, studenti, forze politiche o singoli imputati. Nel processo penale di primo grado contro Montedison, Enichem, Montefibre, e in altri processi relativi al polo chimico di Marghera è stato difensore delle Parti Civili (Medicina Democratica, Associazione Sindacale Lavoratrici/Lavoratori Chimici della Federazione di Venezia), come di altre persone fisiche $^{20}$.
\end{abstract}

\footnotetext{
19 URL:

<http://www.medicinademocratica.org/IMG/pdf/STATUTO_Assoc._MEDICINA_DEMOCRA TICA.pdf $>$ [consultato il 20 agosto 2014].

20 URL: <http://iveser.it/index.php?option=com_content\&task=view\&id=444\&Itemid=62> [consultato il 20 agosto 2014].
} 
Con l'esposto di Scatturin e Bortolozzo, iniziano, da parte di Felice Casson, gli accertamenti preliminari e le prime relazioni tecnico-scientifiche degli esperti nominati dal Procuratore stesso. Nel novembre del 1996 viene dunque depositata dal PM una richiesta di rinvio a giudizio «della quale colpisce una lista infinita di nomi: le persone offese $^{21}$ ». Assieme a loro, chiedono la costituzione di parte civile anche enti pubblici e organizzazioni: comune, provincia, regione, WWF, Greenpeace, Lega Ambiente, associazioni sindacali, Medicina Democratica.

Il processo, iniziato effettivamente il 3 marzo 1997, si è svolto sino alla Cassazione, la cui sentenza fu emanata nel maggio del 2006; l'esito confermava la precedente sentenza d'Appello (2004) e abbandonava definitivamente la motivazione che aveva voluto l'assoluzione di tutti gli imputati in $\mathrm{I}^{\mathrm{o}}$ grado di giudizio. All'epoca della prima sentenza la stampa, che aveva seguito il processo del secolo con attenzione crescente e di pari passo all'aumentare della sensibilità collettiva rispetto al tema, riporta il grido che aveva accompagnato in aula le parole del presidente della Corte ${ }^{22}$ :

\begin{abstract}
Sconcertata la reazione dei presenti nel corso della lettura. Nei cinque minuti che sono serviti al presidente del tribunale, Ivano Nelson Salvarani, per leggere le decisioni dei giudici, nell'aula bunker si è scatenato il finimondo: familiari e dipendenti del Petrolchimico hanno iniziato a urlare "vergogna, vergogna"; il prosindaco di Venezia, Gianfranco Bettin, è scoppiato in lacrime ${ }^{23}$.
\end{abstract}

Quando in Appello e in Cassazione venne ribaltata la sentenza con la condanna di cinque dirigenti Montedison, si parlò non solo di giustizia ma anche di «sconfitta della macchina giudiziaria ${ }^{24}$ ». Molte imputazioni caddero infatti in prescrizione e alle cinque condanne se ne sarebbero aggiunte altre due se Eugenio Cefis e un suo alto dirigente non fossero nel frattempo deceduti.

Il valore del diritto alla salute previsto dall'art. 32 della Costituzione italiana veniva ripristinato. Tra le cinque condanne quella di Emilio Bartalini, responsabile medicosanitario centrale della Montedison, potrebbe risultare la più agghiacciante dato che la struttura sanitaria è preposta alla salvaguardia della salute dei lavoratori. Invece «se

${ }^{21}$ URL: < http://agb.provincia.venezia.it/processocvm/processo.htm > [cosultato il 20 agosto 2014].

22URL:

<http://archiviostorico.unita.it/cgibin/highlightPdf.cgi?t=ebook\&file=/golpdf/uni_2001_11.pd f/o3INTo3A.pdf\&query=processo\%20Montedison\%20Porto\%20Marghera> [consultato il 20 agosto 2014].

${ }_{23}$ URL: < http://www.repubblica.it/online/cronaca/chimico/sentenza/sentenza.html > [consultato il 20 agosto 2014] .

${ }^{24}$ CASSON, Felice, La fabbrica dei veleni cit., p. 334. 
qualcuno lamentava un piccolo fastidio al fegato, i medici di fabbrica ripetevano sempre di bere meno» ${ }^{25}$.

Era iniziata così l'avventura del Petrolchimico: la promessa di uno sviluppo che avrebbe portato benessere a tutti, concentrata nelle mani di pochi; è finita con il fallimento di fronte alla storia e alla legge di quei pochi che avevano avuto tra le mani non solo una promessa ma le vite di molti.

\section{La prova scientifica nel processo penale}

Il processo a Marghera si è giocato soprattutto attorno alle perizie e all'ingente quantità di documentazione, studi, statistiche e indagini volte a individuare o meno un nesso di causa-effetto tra CVM e carcinogenesi. I giudici di $\mathrm{I}^{\mathrm{o}}$ grado nella sentenza hanno dato largo spazio a teorie scientifiche, studi, perizie e in base alle prove acquisite hanno finito per assolvere tutti. Ma il ragionamento seguito è stato in entrambi i due gradi successivi corretto: l'assenza di certezza scientifica era stato il criterio in base al quale deresponsabilizzare i dirigenti della Montedison rispetto alla morte degli operai ma in Appello e in Cassazione i giudici sentenziarono che la responsabilità c'era ed era stata quella di non prevenire i rischi.

Le decisioni prese nel lungo processo a Porto Marghera rientrano in una questione ad oggi ancora aperta che ha come protagonisti avvocati, giudici, scienziati, teorici del processo e riguarda il tema della scienza all'interno del processo penale strettamente connesso a quello delle perizie.

Nel caso del processo ai dirigenti Montedison, per acquisire dati e valutazioni, i giudici dovettero necessariamente nominare dei periti in quanto, per valutazioni che implichino specifiche competenze di ordine tecnico e scientifico non è sufficiente il normale bagaglio di conoscenze del giudice stesso. Recita l'art. 220 comma 1 del Codice di Procedura Penale:

«La perizia è ammessa quando occorre svolgere indagini o acquisire dati o valutazioni che richiedono specifiche competenze tecniche, scientifiche o artistiche ${ }^{26}$.

Nel nostro caso si trattava di risalire ai danni derivanti dall'esposizione a materiali cancerogeni e di ricostruirne i singoli passaggi, tutti di natura chimica e biomolecolare.

Più in generale il giudice deve ricorrere ai periti perché i fatti di cui deve occuparsi non rientrano nelle sue conoscenze e anche l'accusa e la difesa possono far eseguire

\footnotetext{
25 Ibidem, p. 37.

26 URL: < http://www.diritto-penale.it/la-perizia-e-la-consulenza-tecnica-nel-processopenale.html $>$ [consultato il 20 agosto 2014]
} 
ulteriori valutazioni e ricerche ai consulenti tecnici o periti di parte (art. 225 comma 1 cpp).

Tale ricorso alla scienza è dovuto al fatto che «accade sempre più spesso che il giudice debba occuparsi di reati o di illeciti civili che sono stati commessi, per così dire, con mezzi scientifici e che quindi richiedono, per essere accertati, l'impiego della scienza»27. Una ragione ulteriore per ricorrere alla scienza nel processo penale, continua Michele Taruffo, «è data dal diffondersi del convincimento che la conoscenza scientifica rappresenti il paradigma ideale della conoscenza certa e empiricamente dimostrata ${ }^{28}$. Il processo cerca di ricostruire un fatto storico per verificare se e in che modo siano stati commessi reati e la scienza sempre più spesso viene utilizzata a tal fine.

La necessità di prove scientifiche (ancora non importa se perizie o consulenze) implica però l'emergere di due problemi fondamentali: da un lato il giudice «è chiamato a maneggiare strumenti ad alto rischio» dall'altro la scienza «non è nata per l'applicazione processuale. Il sapere scientifico, di per se afinalistico e in perenne evoluzione, si misura con il processo penale orientato invece verso una decisione definitiva»29. Problematico dunque per i giudici districarsi tra leggi scientifiche che potrebbero essere garanzia di verità ma allo stesso tempo sono in continuo cambiamento.

Lo strumento previsto dal sistema italiano (a differenza di quello americano), che il giudice deve utilizzare nel percorrere il labirinto delle prove, è il ragionamento. Vale a dire che anche se le prove dovessero risultare schiaccianti il giudice è tenuto a motivare in maniera esplicita la lettura delle prove stesse e le ragioni delle sue decisioni. I criteri che il giudice segue devono essere presenti nella parte della sentenza dedicata ai motivi di fatto e di diritto in modo tale che nei gradi successivi al primo si possa valutare non l'insieme delle prove ma proprio il ragionamento: «il controllo di legittimità dell'iter logico seguito è affidato alla Corte di Cassazione che scrutina il ragionamento probatorio del giudice di merito in quanto all'accertamento del fatto»30. Ciò è indispensabile a maggior ragione nei processi in cui le prove scientifiche sono

${ }_{27}$ TARUFFO, Michele, Scienza e processo, sub vocem «XXI SECOLO», URL: < http://www.treccani.it/enciclopedia/scienza-e-processo_(XXI-Secolo)/ > [consultato il 20 agosto 2014].

${ }^{28}$ Ibidem.

29 CONTI, Carlotta (a cura di), Scienza e processo penale. Nuove frontiere e vecchi pregiudizi. Milano, Giuffrè, 2011, pp. XIII-XIV.

3o CANZIO, Giovanni, La valutazione della prova scientifica fra verità processuale $e$ ragionevole dubbio, in Scienza e processo penale cit., p. 66. 
determinanti, perché in essi emerge il problema e la difficoltà del giudice di elaborare il suo ragionamento senza farsi condizionare dalla presunta correttezza della scienza.

Un altro problema inerente il rapporto tra scienza e processo penale riguarda un aspetto fondamentale: attraverso le prove scientifiche spesso avviene l'intera ricostruzione dei fatti. Se occorre stabilire l'esistenza di un rapporto causa-effetto (nel caso di Porto Marghera tra sostanza tossica e cancro), si ricorre nei processi al ragionamento definito nomologico-deduttivo che si fonda sul ricorso a una legge generale dotata di fondamento scientifico. Secondo l'avvocato Stella, che molto ha scritto a riguardo e che era il principale difensore dei dirigenti Montedison, per giungere a una conclusione certa bisogna partire proprio da una legge generale da cui poter dedurre se il rapporto causa-effetto si è verificato anche nel singolo caso. Per capire se gli operai di Porto Marghera sono morti a causa del CVM-PVC bisogna partire insomma da una legge approvata dal mondo scientifico e stabilire soprattutto quale fosse la situazione della scienza negli anni in cui i primi tumori iniziavano a fare vittime. Se non ci sono leggi generali cui fare riferimento si può ricorrere a frequenze statistiche così elevate (superiori all'80-90\%) da far ritenere che vi sia un'alta probabilità che, dato un fenomeno $\mathrm{F}$, si sia verificato anche F1 nel singolo caso. Anche sul valore probatorio delle frequenze statistiche vi è una discussione di metodo e sempre Stella, definito «maestro indiscusso della causalità»31, sostiene che quando la probabilità statistica non è elevata i processi non dovrebbero neanche iniziare. È ciò che disse anche nel caso del processo a Marghera. Allo stesso tempo «le frequenze statistiche basse o molto basse sono proprio quelle fornite dalle indagini epidemiologiche sull'esposizione a sostanze tossiche»32 e sarebbe impensabile non istruire quei processi che sulle indagini epidemiologiche si basano: da Porto Marghera al processo Eternit (in questo ultimo caso morirono più tremila persone tra operai, dirigenti, familiari e cittadini). Né si può pensare che l'unico criterio per stabilire se esiste colpa sia quello della causalità: la Corte di Cassazione del processo alla Montedison stabilì che anche se ci fossero stati solo dei dubbi (e ce ne erano molti) sul nesso causa-effetto, bisognava comunque intervenire.

Dunque il punto non è se istruire processi o meno ma risiede nella valutazione delle prove scientifiche da parte dei giudici. Un primo aspetto riguarda il contraddittorio: scrive Paolo Tonini, Ordinario di Diritto Processuale Penale, che il contraddittorio relegato apparentemente solo alla prova dichiarativa si deve e «si può fare anche sulla

${ }^{31}$ CUCCI, Monica, GENNARI, Giuseppe, GENTILOMO, Andrea, L'uso della prova scientifica nel processo penale, Santarcangelo di Romagna, Maggioli, 2012, p. 66.

$3^{2}$ TARUFFO, Michele, Scienza e processo cit., p. 3. 
scienza, valorizzando il confronto tra gli esperti sia nel selezionare i fatti rilevanti, sia nell'applicazione delle leggi scientifiche a quei fatti. [...] In sostanza il contraddittorio va applicato anche alla prova scientifica»33. Evitando così il rischio di sussumere come assolutamente certe valutazioni che hanno base scientifica.

La fase successiva al contraddittorio è quella della formulazione della decisione finale sui fatti: anzitutto la decisione deve essere presa oltre ogni ragionevole dubbio.

Tale principio, come affermato dalla giurisprudenza di legittimità, rappresenta il limite alla libertà di convincimento del giudice, apprestato dall'ordinamento per evitare che l'esito del processo sia rimesso ad apprezzamenti discrezionali, soggettivi e confinanti con l'arbitrio; si tratta di un principio che permea l'intero ordinamento processuale 34 .

A partire da questa base fondante, la scienza potrebbe essere uno strumento efficace per arrivare a sentenziare oltre ogni ragionevole dubbio e proprio per questo l'utilizzazione della scienza nella fase di decisione finale assume un rilievo fondamentale. « $\grave{E}$ questa la fase del processo in cui il giudice si fonda sulle conoscenze scientifiche acquisite al giudizio per accertare, e eventualmente valutare, i fatti della causa» 35 .

Ed è questa la fase in cui il giudice deve fare i conti con il secondo problema dibattuto tra i giuristi: «la perizia è ancora interpretata come una prova del giudice e gode di un credito particolare di per sé»36. Ciò, continua Tonini, «ostacola il contraddittorio» ma inoltre «si ripercuote immediatamente sulla motivazione e induce il giudice ad appiattirsi sulla perizia, che, così, rischia di diventare un nuovo tipo di prova legale»37. Il professore è invece per la neutralità della prova scientifica «che è diventata la più importante nel processo penale» $3^{8}$ e asserisce che essa deve essere considerata al pari delle prove portate dalle parti (le consulenze) perché, a non essere neutrale, è lo scienziato: «In verità, la scelta del metodo scientifico non è mai, in sé,

33 TONINI, Paolo, Dalla perizia "prova neutra" al contraddittorio sulla scienza in Scienza e processo penale cit., p. 4.

34 URL: < http://www.filodiritto.com/articoli/2009/o8/loltre-ogni-ragionevole-dubbio-comeregola-di-giudizio/ > [consultato il 20 agosto 2014].

35 TARUFFO, Michele, Scienza e processo cit., p. 13.

${ }^{6}$ TONINI, Paolo, Dalla perizia "prova neutra" al contraddittorio sulla scienza in Scienza e processo penale, cit., p. 9 .

37 Ibidem.

${ }^{38}$ Ibidem, p. 11. 
neutra; dipende dal singolo esperto, dalla sua competenza, dal laboratorio in cui opera $[\ldots] \gg 39$.

In tale complesso quadro, vale un principio fondamentale, ben esplicitato da Taruffo:

le conoscenze fornite dall'esperto, le sue informazioni, le sue valutazioni e le sue opinioni, per quanto esse siano autorevoli, attendibili e influenti, non possono mai considerarsi vincolanti per il giudice. L'esperto, [...] non può mai sostituirsi al giudice nella formulazione della decisione finale: [...] il giudice conserva intatta la sua discrezionalità nell'accertamento e nella valutazione dei fatti, in base al principio fondamentale del libero convincimento ${ }^{40}$ del giudice stesso, che ormai da tempo sta alla base di tutti gli ordinamenti processuali ${ }^{4}$.

Se però il giudice utilizza i risultati delle prove scientifiche aderendo alle conclusioni formulate dall'esperto (e ciò avviene a maggior ragione se parliamo di perizie), c'è il rischio di una sorta di subordinazione del giudice alle risultanze della prova scientifica e una sostanziale rinuncia dello stesso a esercitare la propria funzione autonoma di controllo. "Il giudice non deve limitarsi a rinviare a ciò che è emerso dalla consulenza tecnica o dalla perizia perché in questo modo egli evita di elaborare la propria motivazione del giudizio ${ }^{42}$.

Facendo il punto della situazione, le sentenze dei giudici all'interno dei processi penali che prevedono prove scientifiche sono minate da: l'idea che la scienza sia fonte di verità praticamente assoluta; il fatto che la prova scientifica sia spesso anche perizia cioè valutazione "superiore" rispetto a quelle di parte.

Alla luce di tale complessa situazione, in che modo si mossero il PM Felice Casson e le parti civili, gli avvocati della difesa e infine i giudici durante il processo a Porto Marghera?

\footnotetext{
39 Ibidem.

${ }^{40}$ L'art. 192 comma 1 del cpp recita: "il giudice valuta la prova dando conto nella motivazione dei risultati acquisiti e dei criteri adottati”.

${ }^{41}$ TARUFFO, Michele, Scienza e processo cit., pp. 14.

42 Ibidem, pp. 14-15.
} 


\section{Dentro le sentenze: l’iter logico dei giudici}

Prima di delineare quali furono i ragionamenti seguiti dai giudici di $\mathrm{I}^{\circ}$, $\mathrm{II}^{\circ}$ e $\mathrm{III}^{\circ}$ grado all'interno del processo a Porto Marghera, si vedano brevemente le scelte dell'accusa e della difesa in tema di prove scientifiche portate in fase dibattimentale.

Felice Casson descrive in Tribunale la linea scelta sin dalle indagini preliminari in questi termini:

[...] è stata sempre l'impostazione dell'accusa, nel senso di avere sempre fornito indicazione ai propri consulenti, coloro che effettuavano materialmente le indagini, di non forzare assolutamente in alcuna maniera la mano, per avere dei dati oggettivi il più possibile. Da potere valutare, potere confrontare al fine di verificare se c'era spazio per effettuare un'indagine e poi un processo penale 43.

La linea della difesa fu impostata differentemente e è riassumibile nell'impossibilità per i dirigenti Montedison di essere a conoscenza del rischio, in quanto la scienza stessa non concordava o non aveva stabilito leggi generali cui attenersi. Basti pensare che dalla difesa fu proposto il Professor Agazzi, Ordinario di Filosofia Teoretica al tempo del processo ma precedentemente Ordinario di Filosofia della Scienza per molti anni; l'avvocato Stella, principale difensore degli imputati, congederà il professore a seguito del suo intervento con la seguente espressione:

Ringrazio il professor Agazzi, mi viene in mente quello che diceva Kant, che gli scienziati sono come dei nomadi che vanno da una città all'altra avendo perso la propria identità e la propria matrice comune. Il professor Agazzi credo che abbia fatto un tentativo di farci ritrovare la matrice comune 44 .

Scienziati nomadi, che hanno perso l'identità: scienze, (in particolar modo ci si riferiva all'epidemiologia), i cui risultati sono tutt'altro che certi.

Tale impostazione non volle affatto suggerire al giudice di svincolarsi dai rischi introdotti dalle prove scientifiche e di maneggiarle con cautela ma dimostrare che non essendoci leggi generali certe non fosse verificato né verificabile dai dirigenti Montedison il nesso causa effetto.

43 URL: < http://ivdi.it/Petrolchimico/home_petrolchimico.htm > [consultato il 20 agosto 2014]. 44 Ibidem. 
Proprio attorno alla dimostrazione dell'esistenza effettiva o non effettiva del rapporto causa-effetto tra sostanza e carcinogenesi ruotano le perizie e gli studi riproposti nella sentenza di $\mathrm{I}^{\circ}$ grado, che seguì la linea della difesa dell'avvocato Stella.

La prima sentenza presenta i risultati degli studi epidemiologici, delle valutazioni di rischio sulla base di modelli matematici, degli studi sui meccanismi molecolari e sulla carcinogenesi. Gli studi epidemiologici hanno portato a inquadrare la tipologia dei tumori (cancro del fegato, del polmone, del cervello, tumori del sistema emolinfopoietico, cirrosi), il periodo in cui gli operai li avrebbero potuto contrarre. Gli studi cui nella sentenza si fa maggior riferimento sono lo Studio Simonato (1991) e il relativo aggiornamento di Ward-Boffetta (2000); gli Studi epidemiologici a Porto Marghera (1991-1995) e il relativo aggiornamento del 1999.

Lo Studio Simonato partiva da studi precedenti sui roditori (Viola- Maltoni) e sull'uomo (Crech-Jones-Wu), iniziò nel 1987 e rappresentava, con i suoi 12.706 casi esaminati, il più ampio modello epidemiologico portato a termine sugli esposti a CVM. A differenza del secondo è stato svolto non solo a Porto Marghera ma anche in altri paesi d'Europa ma entrambi conclusero che l'associazione tra esposizione a CVM e tumore del fegato sussistesse. Gli studi risultarono divergenti riguardo gli altri tre organi bersaglio: il polmone, il cervello e il sistema linfatico. Lo studio Simonato evidenziò che non furono colpiti dal CVM, lo studio a Porto Marghera riscontrò invece come fra gli insaccatori vi fosse stato un incremento significativo del tumore polmonare.

Anche altri studi epidemiologici confermarono una forte associazione tra esposizione lavorativa prima del 1974 e tumori del fegato, nonché l' insorgenza negli insaccatori del tumore del polmone45.

L'aggiornamento da parte di Ward, Boffetta e altri estese lo studio Simonato per gli anni Novanta giungendo agli stessi risultati; venne osservata inoltre l'insorgenza della cirrosi senza evidenziare rischi significativi e anche nel caso del dicloroetano non risultò alcun nesso con le neoplasie gastriche ${ }^{46}$.

Gli studi caso-controllo riguardanti i tumori e le malattie epatiche e polmonari dei lavoratori di Porto Marghera durante gli anni Cinquanta e Sessanta, hanno confermato che il CVM fosse la causa scatenante dei casi di angiosarcoma e epatocarcinoma ${ }^{47}$ e nonostante gli esperti avessero stabilito un nesso cogente tra malattia epatica e esposizione al CVM, il Tribunale si basò sul parere dei periti e dei consulenti della

45 Studi della coorte USA; studi di Waxweiler, Storevedt-Heldaas, Jones, Wu, Comba-PiratsuChellini e del NIOSH.

${ }^{46}$ Studi di Hogstedt (1979); Austin (1983); Olsen (1997); NCI.

47 Studi di Gennaro; Mastrangelo; Martines; Popper; Pinzani . 
difesa concludendo che le epatopatie fossero associate non già all'esposizione a CVM, bensì a consumo alcolico o a epatiti virali. Attraverso la diversa letteratura scientifica cui periti e consulenti della difesa fecero riferimento il Tribunale sentenziò anche che a determinare un aumento del rischio di tumore fossero il sovrappeso corporeo, il diabete, la steatoepatite non alcolica, l'accumulo epatico di ferro e la celiachia. Solamente la Sindrome di Raynaud fu riconosciuta come dovuta a microtraumi causati da sostanze chimiche come il CVM.

Infine vennero presentate e accolte le valutazioni di rischio sulla base di modelli matematici attraverso cui si specificava che sebbene non fosse possibile stabilire livelli sicuri di esposizione, a livelli bassissimi non c'erano rischi significativi per la salute ${ }^{48}$.

Il pubblico ministero nel corso dell'istruttoria dibattimentale mediante i suoi consulenti tecnici introdusse il tema dei meccanismi molecolari attraverso i quali si era visto che il CVM induceva non solo l'angiosarcoma ma anche l' epatocarcinoma49. Il Tribunale però non considerò queste analisi per le sue decisioni finali e ciò fu uno dei motivi per cui il PM ricorse in Appello.

Dunque, passati in rassegna molto sinteticamente gli studi scientifici cui si fece riferimento per la stesura delle sentenze, non rimane che esplicitare i parametri scelti di volta in volta dai giudici per definire o meno l'esistenza della colpa ${ }^{50}$.

La sentenza di $\mathrm{I}^{\circ}$ grado si articola in più di duemila pagine in cui sono dettagliatamente esposte le perizie e gli studi; nelle successive due sentenze sono riportate le doglianze di accusa e difesa che vennero accolte o rifiutate ma soprattutto viene ridiscusso il ragionamento del Tribunale. Esso venne a tal punto modificato nella sostanza che l'assoluzione divenne condanna. Il ragionamento dei giudici di $\mathrm{I}^{\circ}$ grado considerò la colpa non attribuibile perché dalle perizie e dalle prove raccolte, emergeva che la scienza non fosse arrivata a indicare con precisione il rischio del nesso CVMtumore. Nei successivi gradi del processo a Marghera non fu il nesso di causalità, tanto caro all'avvocato della difesa Stella, ad essere determinante nell'attribuzione o meno

${ }^{48}$ Relazioni del prof. Zapponi e dell'Enviromental Protection Agency.

${ }^{49}$ La parte relativa alle perizie e agli studi scientifici si trova integralmente nella sentenza di $\mathrm{I}^{\circ}$ grado scaricabile dal sito e in particolare nella sezione ENICHEM capitolo EPIDEMIOLOGIA e CAUSALITà.

URL: < http://ivdi.it/Petrolchimico/home_petrolchimico.htm > [consultato il 20 agosto 2014]. ${ }^{50}$ La colpa è il criterio di attribuzione soggettiva della responsabilità penale che si contrappone al dolo in quanto è caratterizzato dall' assenza della volontà di alcuno o di tutti gli elementi del fatto tipico. [...] La colpa può essere specifica o generica; la sentenza finale accusò gli imputati di colpa generica perché essa consiste in un comportamento imprudente, un'azione, cioè, che, secondo le massime d'esperienza, non doveva essere compiuta o doveva esserlo in guisa differente; un comportamento non diligente e, cioè, l'omissione di un atto dovuto secondo le prescrizioni delle massime d'esperienza; un'imperizia e, cioè, un comportamento imprudente o non diligente per violazione di regole tecniche da parte di soggetti particolarmente qualificati. URL: <http://www.diritto-penale.it/la-colpa.htm > [consultato il 20 agosto 2014]. 
della colpa e dunque della pena ma il parametro del rischio; per comprendere l'iter logico seguito nei tre gradi di giudizio e le sostanziali differenze, si ripercorre adesso la sentenza di Cassazione: nella parte di fatto e di diritto i giudici riproposero il cardine del ragionamento del Tribunale e concordarono con la Corte d'Appello nel ribaltarne le conclusioni.

Il Tribunale, scrisse la Corte di Cassazione «rileva che la misura della diligenza dovuta [dagli imputatati] è correlata alla prevedibilità dell'evento che deve essere riconosciuta sulla base della migliore scienza e esperienza presenti in un determinato settore e in un preciso momento storico ${ }^{51}$. La prevedibilità deve riguardare un evento «che possa concretamente e effettivamente verificarsi e non già un evento di contenuto generico o realizzabile in via di mera ipotesi. La prevedibilità di un evento può essere formulata solo allorquando [...] sussistano leggi scientifiche di copertura le quali permettano di stabilire che da una certa condotta possano conseguire determinati effetti»52. Dunque, continua la Cassazione, secondo il ragionamento seguito in $\mathrm{I}^{\circ}$ grado: «non esiste responsabilità per colpa quando l'agente non abbia la possibilità di rappresentarsi non tanto gli esatti sviluppi dell'azione lesiva, ma certamente la tipologia delle conseguenze cui il proprio eventualmente negligente operato può dar luogo»53. Infine sempre il Tribunale sostenne che

l'adeguamento del datore di lavoro alle nuove conoscenze scientifiche deve essere tempestivo ma sarà esigibile nel momento in cui le stesse abbiano raggiunto un grado adeguato di consistenza e di solidità, cioè allorquando sia stato conseguito un patrimonio scientifico consolidato, alla luce degli organismi internazionali operanti in materia. Deve quindi escludersi, secondo il Tribunale, l'esistenza della colpa perché l'evento tumore è divenuto prevedibile soltanto nel 1974 e, da quell'epoca in poi, la Montedison adottò tutti gli opportuni interventi per eliminare o ridurre al minimo le esposizioni 54

Il secondo grado di giudizio ribaltò però le suddette conclusioni affermando che

il parametro di valutazione nella colpa è il rischio e non è richiesto che l'agente conosca i meccanismi causali della sostanza. A differenza che nella causalità la

${ }^{51}$ Sentenza presente in estratto in Cass. sez. IV pen. 6 febbraio 2007, n. 4675 in Cassazione penale, 2009.

${ }^{2}$ Ibidem.

53 Ibidem.

54 Ibidem, p. 49. 
prevedibilità va valutata con riferimento alle nozioni conosciute o conoscibili all'epoca in cui la condotta è stata posta in essere55.

Alla luce di questi principi la Corte d'Appello ritenne errato il percorso seguito dalla sentenza di $\mathrm{I}^{\circ}$ grado:

Poiché il parametro della colpa è il rischio, e non l'accertamento condiviso del fondamento scientifico di un'ipotesi, già nel 1969 la Montedison era tenuta ad adottare - anche in base all'art. 2087 del codice civile $5^{6}$ - quegli interventi poi adottati dal 1974 in avanti. All'epoca degli studi del dottor Viola ci si trovava infatti non in presenza di una mera congettura ma di un'ipotesi seria e attendibile anche se da avvalorare con approfondimenti poi effettivamente disposti dalla Montedison (a conferma della serietà dell'ipotesi) con incarico al prof. Maltoni57.

Secondo la Corte d'Appello in presenza di un serio dubbio sulla potenzialità lesiva di una sostanza il rapporto di accertamento è invertito; occorre intanto adottare tutte le misure necessarie per evitare l'esposizione fino a che non venga raggiunta la prova che la sostanza è innocua, secondo il criterio della cosiddetta default opinion. Ne conseguì secondo la Corte d'Appello, la responsabilità per gli omicidi colposi dei lavoratori deceduti per angiosarcoma (in realtà un solo omicidio colposo è ritenuto non ancora prescritto quello di Tullio Faggian). La Corte di Cassazione, concordò con l'iter logico seguito dalla Corte d'Appello e sottolineò come non solo l'art. 2087 fosse stato violato ma anche il d.p.r 303:

Basti pensare che l'art. 20 parla di gas o vapori tossici e il CVM è inserito nella tabella allegata al citato d.p.r 303 riferita alle lavorazioni industriali che espongono all'azione di sostanze tossiche o infettanti o che risultano comunque nocive. Quindi si tratta di una sostanza la cui natura tossica o nociva è riconosciuta dalla legge58!

La nocività del CVM non era da ricercarsi tra le teorie scientifiche ma nella legge italiana e a tale esclamazione, che sembra porre con enfasi una definitiva e radicale distanza dalla sentenza di $I^{\circ}$ grado, seguono le seguenti affermazioni: «le conoscenze rilevanti non sono solo quelle diffuse nella cerchia degli specialisti, e tanto meno le conoscenze avanzate di taluni centri di ricerca, bensì sono le conoscenze che

55 Ibidem, p. 62.

${ }^{56}$ Esso recita che: «L'imprenditore è tenuto ad adottare nell'esercizio dell'impresa le misure che, secondo la particolarità del lavoro, l'esperienza e la tecnica, sono necessarie a tutelare l'integrità fisica e la personalità morale dei prestatori di lavoro».

57 Cass. sez. IV pen. 6 febbraio 2007, n. 4675 cit., p. 62.

${ }^{8}$ Ibidem, p. 277. 
costituiscono un patrimonio diffuso a partire da una certa data»59. Dunque i dirigenti della Montedison sono colpevoli perché dovevano agire e prevenire in base non alle certezze ma alle conoscenze raggiunte, ossia anche solo in base alle ipotesi scientifiche che risalivano addirittura a prima del 1969: «i documenti aziendali Montedison sul finire degli anni '50 riconoscevano la tossicità di queste sostanze; l’imputato Bartalini ha riconosciuto l'epatotossicità del CVM risalente ai suoi studi universitari [...]»60.

Quindi

$$
\begin{aligned}
& \text { ben da prima dell'epoca della contestazione (1969) dovevano già ritenersi } \\
& \text { prevedibili gravi danni alla salute dei lavoratori esposti al CVM e doveva ritenersi } \\
& \text { sorto l'obbligo [...] di adottare le cautele necessarie [...] perché la tutela della salute } \\
& \text { umana costituisce obbligo primario di salvaguardia di un diritto costituzionalmente } \\
& \text { protetto (art. } 32 \text { della costituzione) }{ }^{61} \text {. }
\end{aligned}
$$

In $I^{\circ}$ grado l'attenzione dei giudici si era invece focalizzata sulle conoscenze scientifiche, quasi affidando ad esse il compito di giudicare se le leggi italiane fossero state rispettate o meno. A proposito del rapporto tra scienza e giurisprudenza profilatosi in $\mathrm{I}^{\mathrm{o}}$ grado la Corte di Cassazione sentenziò allora che

\begin{abstract}
la soglia oltre la quale l'agente può prevedere le conseguenze lesive della sua condotta, non è costituita dalla certezza scientifica ma dalla probabilità o anche dalla sola possibilità che queste conseguenze si producano. [...] Evidente è quindi l'erroneità della tesi del Tribunale secondo cui, per far sorgere l'obbligo prevenzionale, occorre fare riferimento al patrimonio scientifico consolidato quale criterio per imporre l'adozione della regola cautelare ${ }^{62}$.
\end{abstract}

Il dovere di tenere una condotta responsabile da parte dei dirigenti nei confronti della salute dei lavoratori esiste prima e a prescindere dalla certezza scientifica. Sono solamente i princípi di responsabilità e prevenzione a dover guidare le azioni di questi individui e di chi deve giudicare tali azioni.

\footnotetext{
59 Ibidem, p. 282.

${ }^{60}$ Ibidem, p. 286.

${ }^{61}$ Ibidem, p. 287.

62 Ibidem, p. 290.
} 


\section{Conclusioni}

Si vuol concludere questa analisi, che risente dei limiti imposti dall'ingente quantità di documenti da consultare e della loro specificità tecnica, con le parole di Gianfranco Bettin, uno dei protagonisti di questa vicenda, ringraziandolo per l'intervista rilasciatami.

Porto Marghera doveva rappresentare lo sviluppo capitalistico di Venezia, Lei ha scritto in Petrolkiller che è stata comunque una straordinaria avventura industriale e scientifica oltre che umana. Ad una decina d'anni dalla sentenza definitiva, come rivive il disastro causato dalle industrie e quell'avventura?

[...] Si può anche mettere nel conto che un secolo e più fa quando si è deciso l'insediamento, non ci fossero i criteri che abbiamo noi oggi, oppure si può anche capire l'ingenuo entusiasmo in cui un po' tutti, compresi i lavoratori, hanno accettato lo sviluppo industriale come elemento di progresso e anche di propria personale emancipazione da una condizione di povertà; si può persino capire la resistenza nel cogliere gli elementi distruttivi e persino devastanti che quel modello aveva in sé da parte di chi ne era beneficiato secondo la logica di prima: l'idea del progresso, l'emancipazione dalla povertà... se ne sentiva beneficiato ma ne era anche vittima come abbiamo scoperto ben prima del processo ma sancito dal processo. Questi schemi mentali, culturali, anche politici, li si può capire ma per esempio è impossibile assolvere chi, sapendo che un certo modo di organizzare la produzione e di esporre i lavoratori ai suoi effetti, aveva conseguenze anche letali, ha nascosto. Mentre appunto il discorso di prima per me è accettabile sulla base del quadro storico, degli elementi culturali e anche delle forme politiche di allora, quando invece queste cose sono emerse, attestarsi sulla difesa secca con qualche aggiustamento del modello e niente più, quando erano evidenti i danni provocati direttamente sui lavoratori, per me è inaccettabile. Penso che questi dieci anni o più che ci separano dalla sentenza abbiano consentito di digerire un poco questi elementi e che oggi si possa parlare a mente più fredda.

Secondo Lei, se posso parlare di una persona scomparsa, Maltoni come ricorderebbe quel periodo? Con amarezza?

Un po' sì. Io l'ho visto qualche volta. Mi sembrava una persona sicura di sé, delle proprie opinioni e della correttezza del proprio agire quindi in questo senso tranquillo 
con se stesso. Però abbastanza amareggiato col resto del mondo scientifico. Proprio per questa vicenda. Non potrei dire di averlo sentito dire cose pesanti o meglio, cose pesanti sì ma non dirette del tipo: sono venduti! Però una chiara consapevolezza della dipendenza del mondo scientifico dai finanziamenti dell'industria chimica, per il legame che ha con l'industria farmaceutica e medica in generale, certamente l'aveva e l'ha detto.

L'analisi del processo a Marghera qui presentata ha voluto porre in evidenza il rapporto tra scienziati, scienza, dirigenti delle imprese e applicazione della legge. La sentenza finale ha sancito la vittoria di quest'ultima, precedentemente messa in pericolo. Ma il processo non ha riportato in vita i lavoratori morti nelle fabbriche, vittime del tempo utilizzato per cercare di subordinare fino all'ultimo, la salute degli uomini alle conoscenze scientifiche. L'incertezza della scienza, che ha bisogno dei suoi tempi per arrivare a dei risultati concreti non può divenire incertezza della vita, giustificazione della morte. Se ciò avviene quella stessa subordinazione non deve essere estesa tra chi ha il compito di ristabilire la giustizia attraverso la legge. 


\section{* L'autore}

Marina de Ghantuz Cubbe ha conseguito la laurea triennale in Lettere moderne presso

l'Università la Sapienza. Studia Scienze storiche presso l'Università di Bologna con particolare interesse per la storia e il diritto del lavoro. Attualmente collabora con l'associazione Articolo 21. URL: < http://www.studistorici.com/progett/autori/\#deGhantuzCubbe >

\section{Per citare questo articolo:}

de GHANTUZ CUBBE, Marina, "La scienza nel processo penale: Porto Marghera», Diacronie. Studi di Storia Contemporanea : Il diritto militante, 29/12/2014,

URL:< http://www.studistorici.com/2014/12/29/de-ghantuz-cubbe_numero_20/ >

Diacronie Studi di Storia Contemporanea $\beta$ www.diacronie.it

Risorsa digitale indipendente a carattere storiografico. Uscita trimestrale. redazione.diacronie@hotmail.it

Comitato di redazione: Jacopo Bassi - Luca Bufarale - Elisa Grandi - Deborah Paci - Fausto Pietrancosta - Matteo Tomasoni - Luca Zuccolo

Diritti: gli articoli di Diacronie. Studi di Storia Contemporanea sono pubblicati sotto licenza Creative Commons 25 Possono essere riprodotti a patto di non modificarne i contenuti e di non usarli per fini commerciali. La citazione di estratti è comunque sempre autorizzata, nei limiti previsti dalla legge. 